\title{
Performance Modeling of Optical-Burst Switching With Fiber Delay Lines
}

\author{
Xiaomin Lu, Student Member, IEEE, and Brian L. Mark, Member, IEEE
}

\begin{abstract}
We develop analytical models to evaluate the performance of optical-burst switch (OBS) architectures employing fiber delay lines (FDLs) as optical buffers to reduce burst-loss probability. The performance of such architectures cannot be captured accurately using traditional queueing models, since FDLs behave fundamentally differently from conventional electronic buffers. We formulate a Markovian model to evaluate the system performance when the burst-arrival process is Poisson and the burst lengths are exponentially distributed under an idealized model of FDL behavior. The model accurately captures both the balking and deterministic delay properties of FDLs, but the complexity of the model makes it infeasible for solving problems of practical interest. By considering approximations of the model in the regimes of short and long FDLs, we develop relatively simple closed-form expressions that can be used for dimensioning OBS architectures. We also extend the approximate model to include the impact of FDL delay granularity. We present numerical results that validate our modeling approach and demonstrate that significant performance gains in optical-burst switching are achievable when FDLs are employed as optical buffers.
\end{abstract}

Index Terms-Buffers, Markov processes, optical switches, packet switching, traffic control.

\section{INTRODUCTION}

B URST switching is a hybrid form of packet switching and circuit switching that has recently been proposed as a suitable broadband switching paradigm in the optical domain (cf. [1], [2]). The basic transmission unit is a variable-length "burst," which is a concatenation of multiple packets sharing a common source-destination pair in the core network. As in circuit switching, the link bandwidth is divided into smaller capacity channels. In optical-burst switching over wavelength-division multiplexing (WDM) networks, the bursts are transmitted over wavelength channels. The aggregation of packets into longer bursts is performed by switches and multiplexers at the edge of the network. The longer bursts transmitted over wavelength channels alleviate the switching-time requirements of optical-burst switches (OBSs) relative to optical-packet switches.

In optical-burst switching, each burst is transmitted in the form of two components, a small control or burst header packet (BHP) and a data burst (DB), which are transmitted

Paper approved by I. Andonovic, the Editor for Optical Networks and Devices of the IEEE Communications Society. Manuscript received August 15, 2003; revised January 15, 2004 and May 15, 2004. This work was supported by the National Science Foundation under Grant ACI-0133390. This paper was presented in part at the 10th IEEE/ACM International Symposium on Modeling, Analysis, and Simulation of Computer and Telecommunications Systems, Fort Worth, TX, October 2002, and in part at the IEEE International Conference on Communications, Anchorage, AK, May 2003.

The authors are with the Department of Electrical and Computer Engineering, George Mason University, Fairfax, VA 22030 USA (e-mail: xlu@gmu.edu; bmark@gmu.edu).

Digital Object Identifier 10.1109/TCOMM.2004.838731 over separate wavelength channels. The BHP is transmitted in advance of the DB, and is switched electronically at each intermediate switch node. Information contained in the BHP is used to reserve a wavelength channel for the ensuing DB. By properly choosing an offset time between the BHP and the $\mathrm{DB}$, a scheduling algorithm can guarantee that an outgoing wavelength channel is set up by the time the DB arrives at an intermediate switch node. In such a one-way reservation scheme, the end-to-end latency for burst transmission is approximately one round-trip time less than in optical-circuit switching.

The design and performance evaluation of OBSs have been active topics of research in the last few years (cf. [1]-[5]). The concept of burst switching is actually not new (cf. [6]), although the application to the optical domain is relatively recent. Optical-burst switching is a promising candidate for a core IP-over-WDM switching architecture, but a fundamental open problem is how to handle burst contentions that occur when two or more incoming bursts are directed simultaneously to a common output line. Two basic contention resolution approaches that have been considered involve the use of multiple wavelengths and/or fiber delay lines (FDLs). When two bursts contend for the same output line, they may be scheduled for transmission on different wavelengths on the output line. FDLs provide a means to resolve contentions that cannot be resolved by exploiting the wavelength dimension. For example, when fewer than two unscheduled wavelengths are available, an FDL may be used to delay the transmission of one of the contending bursts. It is important to note that the combination of full wavelength conversion and optical buffering can reduce the burst-blocking probability, but does not eliminate blocking altogether.

In this paper, we develop new analytical models to evaluate the performance of optical-burst switching with FDLs. The goal of this paper is to gain insight into the performance impact of FDLs and to develop numerical approximations that can be used to design and dimension OBSs employing FDLs. The performance evaluation of FDLs raises some interesting modeling issues, because the behavior of FDLs is rather different from conventional electronic buffers. An electronic buffer can accept an incoming burst if sufficient space exists in the buffer to accommodate the length of the burst. On the other hand, an FDL can only provide a deterministic delay to an incoming burst. Moreover, the burst must be dropped if the maximum delay provided by the FDL is not sufficient to avoid contention with a burst that is currently being transmitted on a given output line. We refer to this phenomenon as the balking property of FDLs. Unlike earlier work in this area, our performance model captures both the deterministic delay and balking properties of FDLs. ${ }^{1}$

\footnotetext{
${ }^{1}$ Earlier versions of this work were presented in [7] and [8].
} 
As in the earlier work in this area (cf. [1], [3], and [9]), we shall assume that the burst-arrival processes are Poisson and the burst lengths are exponentially distributed. These assumptions have greater validity when the burst lengths are relatively long, in the regime where the performance of burst switching approaches that of circuit switching. The assumptions of Poisson call arrivals and exponentially distributed call-holding times are well accepted as reasonable models for circuit-switched networks, and provide a foundation for basic teletraffic theory. When the burst lengths are shorter, i.e., when the performance of burst switching approaches that of packet switching, it is well known that Poisson/exponential modeling assumptions break down. Nevertheless, our models provide valuable insight into the performance characteristics of optical-burst switching with FDLs and provides a solid basis for further investigations using more realistic arrival-process models and burst-length distributions.

There have been relatively few papers that model the impact of FDLs in OBSs. Turner [1] applied an $M / M / k / D$ queueing model to study the performance of optical-burst switching with FDLs. Based on the $M / M / k / D$ queueing model, Yoo et al. [3] obtained upper and lower ${ }^{2}$ bounds of the burst-loss probability for an optical-burst switching architecture employing FDLs. However, their model (and also that of [1]) fails to capture the deterministic delay and balking characteristics of FDLs. Rather, the behavior of FDLs is essentially approximated by that of conventional buffers. The investigation of the performance impact of FDLs in [3] was carried out mainly via computer simulation. Fan et al. [9] proposed a queueing-based model to improve upon the lower bound of [3]. However, their model fails to capture the balking property of FDLs, i.e., a burst must be discarded if its expected waiting time is longer than the maximum delay provided by all available FDLs. Callegati [10] applied a queueing model with balking to study the impact of choosing a unit delay in FDLs restricted to multiples of the unit delay.

The main contribution of this paper is an analytical approach to characterize the behavior of optical-burst switching with FDLs. Our modeling approach provides a framework for deriving accurate approximations for FDL performance that can be computed efficiently. Our numerical results suggest that FDLs can reduce the burst-loss probability at a switch by two to three orders of magnitude with relatively modest requirements on the maximum FDL length. Such reductions in burst-loss probability at individual burst switches imply even more dramatic reductions in packet-loss probability in the core network. Therefore, the judicious use of FDLs can significantly improve the performance of core WDM networks based on optical-burst switching.

The remainder of the paper is organized as follows. Section II describes the basic optical-burst switching architecture and scheduling mechanisms that are assumed in this paper. In Section III, we develop a new performance model for optical-burst switching with FDLs under the idealization that each FDL can assume a continuous-valued variable delay. The model captures

\footnotetext{
${ }^{2} \mathrm{As}$ indicated in [3], the lower-bound result is not a true lower bound in all cases.
}

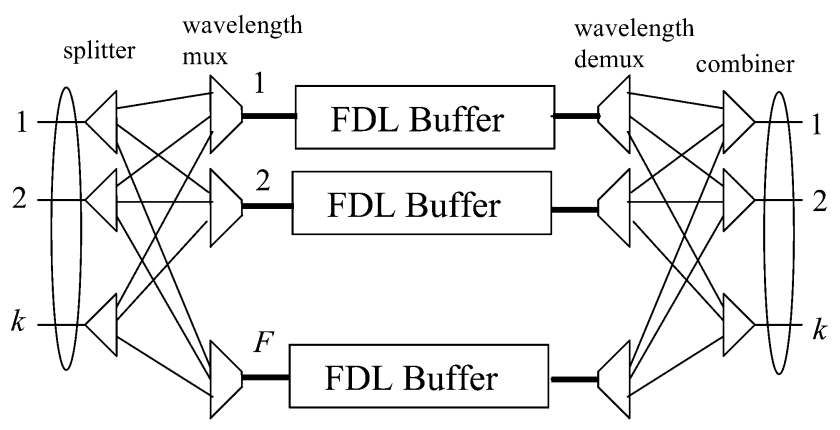

Fig. 1. Structure of the optical buffer at an output port in the data plane of an OBS switch.

the performance impact of FDLs, but is computationally difficult to evaluate numerically when the number of wavelengths in the system is large. Hence, in Section IV, we derive approximations that can be computed efficiently, yet retain the salient performance characteristics of FDLs. In Section V, we extend the approximate FDL models to capture the impact of unit FDL granularity. Numerical results validating our proposed model and approximations are presented in Section VI. Finally, the paper is concluded in Section VII.

\section{OPTICAL-Burst SWITCHING WITH FDLS}

In this section, we describe a generic optical-burst switching architecture that is the basis for our performance analysis. For more detailed discussions of the architectural and implementation issues of OBSs, the reader is referred to [2], [3], and [4].

\section{A. OBS Architecture}

In the basic OBS architecture, each input and output link carries a set of wavelength channels, one of which is a control channel, the rest being data channels. DBs are carried on the data channels, which are switched in the data plane of the OBS architecture. BHPs carried on the control channels are converted to the electronic domain and processed in the switch control unit. The control information stored in the BHPs is used to determine how the DBs are switched in the data plane of the OBS.

Following [4], we shall assume that the data plane of the OBS consists of an ideal $N k \times N k$ nonblocking optical-switching matrix with output buffering, where $N$ is the number of input/output ports, and $k$ is the number of wavelength channels per optical fiber. Fig. 1 shows the basic configuration of an output buffer at an output port of the switching matrix. We shall assume that full wavelength-conversion capability is available at each output port, and that optical buffering is provided in the form of FDLs.

The FDL buffer can be designed in several ways. The fixeddelay FDL buffer illustrated in Fig. 2 (cf. [3], [4]) consists of a splitter which divides the input optical signal into $n$ replicated signals, each of which passes through an FDL with a different delay value ranging from zero to $B=(n-1) b$ in increments of $b$. The wavelength-sensitive multiplexer at the output of the fixed-delay FDL buffer generates an output signal from $k$ wavelength channels, selected (by the control unit) from among the $n$ input signals, each of which consists of $k$ wavelength channels. 


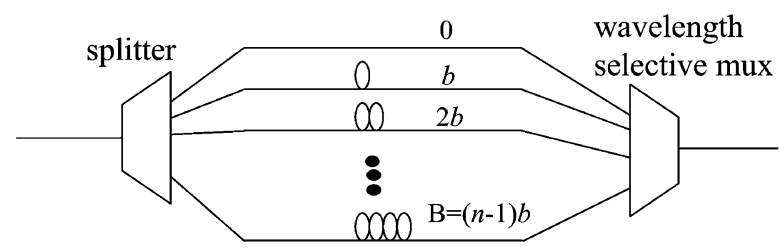

Fig. 2. Fixed-delay FDL buffer.

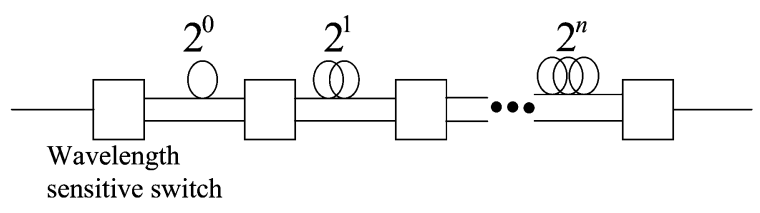

Fig. 3. Variable-delay FDL optical buffer.

The variable-delay FDL buffer shown in Fig. 3 consists of a set of $n$ wavelength-sensitive $2 \times 2$ switches connected by two lines, one with zero delay and the other with a delay of $b$, arranged in a multistage feedforward configuration (cf. [3], [11], [12]). The variable-delay FDL buffer provides a delay for each of the $k$ wavelengths ranging from zero to $B$ in increments of $b$. In combination with the two stages of wavelength converters, both types of optical buffer effectively provide $k$ virtual buffers corresponding to each physical delay line, for a total of $m=k F$ virtual buffers. Here, the term virtual buffer denotes a physical delay line associated with a given wavelength. The variable-delay FDL buffer is more effective than the fixed-delay FDL buffer, because all $m$ virtual buffers are capable of providing a variable delay from 0 to $B$. In effect, the variable-delay FDL can accommodate up to $m$ bursts at a given time instant, provided that each burst requires a delay no more than $B$. On the other hand, the fixed-delay FDL is simpler and less costly to implement.

In our performance model, we shall assume that variabledelay FDL buffers are employed as optical buffers. When $b$ is small or $k$ is large, the two types of FDL buffers have similar performance. To keep the model tractable, in Section III, we shall assume an idealized model in which each of the $F$ variable delay lines can provide any real-valued delay value in the range 0 to $B$. In practice, the performance impact of the delay increment $b$ is nonnegligible, and we shall discuss how the model can be extended to include the effect of $b$ in Section V.

\section{B. OBS Signaling}

In the control plane, each BHP is transmitted $\delta$ seconds ahead of the ensuing DB. The time value $\delta$ is called the base offset time. If a BHP arrives at the switch at time $t$, then the corresponding DB will arrive at the switch at time $t+\delta$. We shall assume that the BHP contains the length of the associated DB and the offset time between the BHP and DB. Upon arrival of the BHP, the arrival and departure times of the ensuing DB can be determined. Thus, an outgoing wavelength channel can be reserved for the DB for precisely its duration. Such a scheme is referred to as the Just-Enough-Time (JET) signaling protocol [2], [13]. In JET, both the arrival and the departure times of a DB are known. As a result, by reserving a specified wavelength only for the duration for the incoming burst, the wavelength utilization is higher for JET than for other protocols [5]. In this paper, we shall assume that the JET signaling protocol is employed.

\section{Wavelength Scheduling and FDL Reservation}

The arrival of the BHP at a switch initiates a process whereby an attempt is made to reserve an outgoing wavelength channel on which to transmit the ensuing DB. The basic reservation scheme consists of two phases: wavelength reservation and FDL buffer reservation. During the wavelength-reservation phase, the scheduler first checks the reservation schedules of the $k$ wavelength channels in the output port. Various wavelength scheduling policies have been proposed in the literature [2], [4]. The latest available unscheduled channel (LAUC) and LAUC with void filling (LAUC-VF) algorithms are two examples.

In the LAUC algorithm, the scheduler maintains for wavelength $i(1 \leq i \leq k)$ the current unscheduled time, $U_{i}$, which represents the earliest time after which no DB transmissions are scheduled on the wavelength. Upon arrival of a BHP, the arrival and departure times of the ensuing DB are computed by the scheduler as $T_{s}$ and $T_{d}$, respectively. Among all of the wavelength channels for which $U_{i} \leq T_{s}$, a channel $i$ with the minimum value of $T_{s}-U_{i}$ is reserved for the DB. If no such channel exists, the virtual buffer-reservation phase begins. In this case, the channel $i$ with the minimum value of $U_{i}$ is considered. If $U_{i}-T_{s} \leq B$, then a virtual buffer with delay value $\left\lceil\left(U_{i}-T_{s}\right) / b\right\rceil b$ is reserved if one is available. In this case, the DB is scheduled for transmission on wavelength $i$ at time $T_{s}+\left\lceil\left(U_{i}-T_{s}\right) / b\right\rceil b$. The value of $U_{i}$ is updated accordingly. Otherwise, if no virtual buffer with the appropriate delay value is available, the burst is dropped.

The LAUC-VF algorithm is similar to LAUC, except that the voids or gaps that may occur between scheduled DBs can be filled by newly arriving DBs. Voids may be generated if bursts have different values of the offset time between the BHP and DB. Voids may also occur due to the granularity of the unit FDL delay $b$. Although the LAUC-VF algorithm can achieve higher utilization than LAUC, LAUC-VF is more complicated to implement because the scheduler must maintain knowledge of the unused voids between DB reservations. In this paper, we shall assume that the simpler LAUC algorithm is used for wavelength and virtual-buffer reservation.

Based on the wavelength reservation and the FDL reservation mechanisms under the LAUC algorithm, the scheduler computes the minimum waiting time $W$ for the blocked DB during the wavelength-reservation phase. If $W<B$ and there is a virtual buffer available, the corresponding FDL and the wavelength will be reserved for the DB. In addition, the waiting time of the DB is equal to the remaining busy duration of the wavelength, i.e., the sum of the residual time of the burst currently in service and the durations of all other bursts that have already been scheduled.

\section{MARKOVIAN MODEL}

We formulate a Markovian model to characterize the performance of an OBS switch architecture employing FDLs, as discussed in Section II. We assume that the destination output port 
of a given arriving burst is uniformly distributed. Thus, it suffices to model the behavior of a typical output port of the optical switching matrix. Each output port consists of $k$ wavelength channels. Thus, each physical FDL can provide up to $k$ virtual buffers, one corresponding to each wavelength. We shall assume that the total number of virtual buffers is given by $m=k F$. The JET signaling protocol and the LAUC scheduling algorithm (cf. Section II) are used to schedule DBs for transmission on outgoing wavelength channels.

Bursts are assumed to arrive according to a Poisson process with a mean rate of $\lambda$ bursts/second. The duration of a DB is an exponentially distributed random variable with a mean of $1 / \mu \mathrm{s}$. We shall further assume that the variable-delay FDLs are employed and are capable of providing any real-valued delay in the range 0 to $B$. The base offset time between the BHP and DB is assumed to be constant among all bursts. We remark that under these last two assumptions, the LAUC and LAUC-VF scheduling algorithms are equivalent. In Section V, we shall relax the idealized assumption of continuous-valued FDL delay.

As mentioned in Section II, there is a fixed offset time of $\delta$ between the BHP and the associated DB. Thus, the scheduled starting time $T_{s}$ of a DB whose BHP arrived before time $t$ satisfies $T_{s}<t+\delta$. However, the DB will be blocked if one or more DBs are scheduled at time $T_{s}$ or later. An idle virtual buffer can be reserved for a blocked DB, as long as its waiting time is less than the maximum FDL delay $B$ that can be provided by the system. Thus, the number of busy virtual buffers can be considered a state variable of the system.

We shall characterize the overall system behavior by a multidimensional continuous-time Markov chain (CTMC). Let $n_{i}$ denote the number of DBs assigned to wavelength $i$, and let $s$ denote the number of busy virtual buffers at time $t+\delta$, where $n_{i} \geq 0$ and $0 \leq s \leq m$. Of the DBs assigned to wavelength $i$ on an output link, at most one can occupy the wavelength channel. The remaining DBs are delayed by the virtual FDL buffer associated with wavelength $i$. When a DB passes through a virtual FDL buffer, it occupies the virtual buffer for a time equal to the duration of the DB.

The system state can be characterized by the tuple $\left(n_{1}, n_{2}, \ldots, n_{k}, s\right)$. For ease of presentation, ${ }^{3}$ we shall assume that the number of wavelengths $k=2$. For $k=2$, the current system state is denoted by $\left(n_{1}, n_{2}, s\right)$. Two types of transitions are possible from this state: $\lambda$-type transitions and $\mu$-type transitions. The $\lambda$-type transitions occur when a DB arrives at the switch. The $\mu$-type transitions are due to DB departures from the virtual buffer or the output port. To make the problem tractable, we assume that $\mu$-type transitions due to DB departures from the FDLs are independent of $\mu$-type transitions due to the DB departures from the output port. According to the values of $n_{1}$ and $n_{2}$, we have the following four cases.

- $n_{1}=0, n_{2}=0$. In this state, there is no burst loss, since the two wavelengths are idle. Therefore, $\lambda$-type transitions lead to the next states of $(1,0, s)$ and $(0,1, s)$ with rates $\lambda / 2$. We assume that the two types of transitions

\footnotetext{
${ }^{3}$ The generalization to $k>2$ is relatively straightforward, and is omitted here for brevity.
}

are equally probable. The $\mu$-type transition rate to state $(0,0, s-1)$, if $s>0$, is $s \mu$.

- $n_{1}=0, n_{2}>0$. In this case, no blocking occurs. The next state due to a $\lambda$-type transition is $\left(1, n_{2}, s\right)$, since the first wavelength is idle. The transition rate is $\lambda$. The $\mu$-type transitions can lead the system to state $\left(0, n_{2}-1, s\right)$ or state $\left(0, n_{2}, s-1\right)$ (if $\left.s>0\right)$. The transition rates to these states are $\mu$ and $s \mu$, respectively.

- $n_{1}>0, n_{2}=0$. Similar to the previous case, $\lambda$-type transitions lead to state $\left(n_{1}, 1, s\right)$ with rate $\lambda$, and $\mu$-type transitions lead to state $\left(n_{1}-1,0, s\right)$ or state $\left(n_{1}, 0, s-1\right)$ (if $s>0$ ), with rates $\mu$ and $s \mu$, respectively.

- $n_{1}>0, n_{2}>0$. In this last case, an arriving DB is blocked in the wavelength-reservation phase, since both wavelengths are busy. The $\mu$-type transitions can lead to states $\left(n_{1}-1, n_{2}, s\right),\left(n_{1}, n_{2}-1, s\right)$, and $\left(n_{1}, n_{2}, s-1\right)$ (if $s>0$ ), with rates of $\mu, \mu$, and $s \mu$, respectively. The next possible states due to $\lambda$-type transitions are $\left(n_{1}+\right.$ $\left.1, n_{2}, s+1\right)$ and $\left(n_{1}, n_{2}+1, s+1\right)$, depending on which wavelength is used.

Let $B_{i}$ denote the remaining busy duration of wavelength $i$ at time $t+\delta$, when the system state is $\left(n_{1}, n_{2}, s\right)$. According to the LAUC scheduling algorithm, the next state due to a $\lambda$-type transition is $\left(n_{1}+1, n_{2}, s+1\right)$ if $B_{1}$ satisfies $B_{1}<B$ and $B_{1}<B_{2}$, and $s<m$. Therefore, the transition rate to state $\left(n_{1}+1, n_{2}, s+1\right)$ when $s<m$ is given by

$$
\begin{aligned}
& \lambda\left[\operatorname{Pr}\left\{B_{1}<B_{2}<B\right\}+\operatorname{Pr}\left\{B_{1}<B<B_{2}\right\}\right] \\
& \quad=\lambda\left[\left(1-E_{n_{1}}\right)\left(1-E_{n_{2}}\right) F_{n_{1}, n_{2}}+\left(1-E_{n_{1}}\right) E_{n_{2}}\right]
\end{aligned}
$$

where $E_{j}$ denotes the probability that an arriving DB on a given wavelength will be discarded when $j$ DBs are scheduled ahead of it, and $F_{i j}$ denotes the probability that the waiting time on wavelength 1 will be less than that of wavelength 2 , given that $i$ and $j$ DBs on wavelengths 1 and 2, respectively, are scheduled ahead of the arriving DB. Similarly, the transition rate to state $\left(n_{1}, n_{2}+1, s+1\right)$ when $s<m$ is given by

$$
\begin{aligned}
& \lambda\left[\operatorname{Pr}\left\{B_{2}<B_{1}<B\right\}+\operatorname{Pr}\left\{B_{2}<B<B_{1}\right\}\right] \\
& \quad=\lambda\left[\left(1-E_{n_{2}}\right)\left(1-E_{n_{1}}\right) F_{n_{2}, n_{1}}+E_{n_{1}}\left(1-E_{n_{2}}\right)\right] .
\end{aligned}
$$

It remains to obtain expressions for $E_{j}$ and $F_{i j}$. Consider a BHP arriving at the system at time $t$. The blocking status of the ensuing DB for a given wavelength depends on the reservation schedule of the wavelength from time $t+\delta$ onwards. Let $R_{0}$ denote the residual service time of the DB being served at time $t+\delta$, and suppose that $j-1$ other DBs have been scheduled and are waiting for service. The blocking probability of the DB arriving at time $t+\delta$ is given by

$$
E_{j}=\operatorname{Pr}\left\{R_{0}+\sum_{i=1}^{j-1} X_{i}>B\right\} .
$$

Using the memoryless property of exponentially distributed random variables, the above blocking probability is equivalent to

$$
E_{j}=\operatorname{Pr}\left\{\sum_{i=0}^{j-1} X_{i}>B\right\}
$$


which can be expressed as

$$
E_{j}=e^{-\mu B} \sum_{n=0}^{j-1} \frac{(\mu B)^{n}}{n !} .
$$

We can see from (4) that the blocking probability is the complementary distribution of a $j$-stage Erlangian random variable, where $j$ is determined by the number of DBs that have reserved the wavelength at or after time $t+\delta$. The probabilities $F_{i j}, i, j \geq$ 0 , can be expressed as follows:

$$
F_{i j}=\operatorname{Pr}\left\{\sum_{l=1}^{i} X_{l}<\sum_{l=1}^{j} Y_{l}\right\}
$$

where $\left\{X_{l}\right\}_{1 \leq l \leq i}$ and $\left\{Y_{l}\right\}_{1 \leq l \leq j}$ are independent sequences of independent, exponentially distributed random variables with common parameter $\mu$. Here, $i$ and $j$ refer to the number of residual stages on the two wavelengths. It can be shown that

$$
F_{i j}=\sum_{n=0}^{j-1}\left(\begin{array}{c}
n+i-1 \\
n
\end{array}\right) \frac{1}{2^{n+i}} .
$$

Let us denote the steady-state probability that the system is in state $(i, j, l)$ by $\pi_{i j l}$. The corresponding burst-loss probability is given by

$$
p_{i j l}=E_{i} E_{j}+\left(1-E_{i} E_{j}\right) \delta_{l m}
$$

where $\delta_{i j}=1$ if $i=j$ and $\delta_{i j}=0$, otherwise. The overall burst-loss probability can then be computed as

$$
\beta=\sum_{i=0}^{\infty} \sum_{j=0}^{\infty} \sum_{l=0}^{m} \pi_{i j l} p_{i j l} \triangleq \operatorname{Markov}(k, \rho, F, \mu B)
$$

where we have introduced the notation $\operatorname{Markov}(k, \rho, F, \mu B)$ to represent the burst-loss probability under the Markovian model as a function of the parameters $k, \rho, F$, and $\mu B$.

The Markovian model can be solved using state-truncation techniques. By iteratively increasing the state space, the burstloss probability can be computed to any desired degree of accuracy. However, the analytical model is not computationally efficient for larger values of $k$, since the dimension of the state space grows with $k$. Nevertheless, the model provides insight into the performance characteristics of OBS with FDLs and provides a basis for deriving simpler analytical approximations to be discussed in the next section.

\section{ASYMPTOTIC APPROXIMATION MODELS}

We shall derive approximations of the general performance model developed in Section III that are more amenable to computation for arbitrary values of $k$. Denote by $A_{W}$ the event that a $\mathrm{DB}$ is blocked in the wavelength-reservation phase. Let $W$ be the waiting time, and $A_{F}$ be the event that the DB is blocked in the FDL reservation phase. Denote the burst-loss probability during the wavelength-reservation phase by $b_{W}=$ $\operatorname{Pr}\left\{A_{W}, W>B\right\}$. Let the loss probability of the burst during the FDL reservation phase be denoted by $b_{F}=\operatorname{Pr}\left\{A_{W}, W \leq\right.$ $\left.B, A_{F}\right\}$. The burst-loss probability is then given by

$$
\beta=b_{W}+b_{F} .
$$

When $B$ is close to zero, we would expect that $b_{F} \ll b_{W}$, since in this case, it is unlikely for a blocked burst to find an available FDL capable of providing a sufficiently long delay. As a result, burst losses during the wavelength-reservation phase play the main role, and for small $B$, we have $\beta \approx b_{W}$. In this case, the balking property of the FDLs plays the dominant role. Conversely, when $B \rightarrow \infty$ and $m>k$, we expect that $b_{W} \ll b_{F}$, since almost all blocked bursts can obtain an available FDL. Therefore, for large $B$, we have $\beta \approx b_{F}$. In this case, the delay characteristic of FDLs dominates. Hence, we shall consider approximations for the burst-loss probability in the regimes of short and long FDLs.

\section{A. Balking Model for Short FDLs}

In the regime of short FDLs, i.e., $B \approx 0$, it is more likely for a burst to be blocked, because the waiting time $W$ is greater than $B$, than for the burst to be blocked because there are not enough available FDLs. This suggests that in the regime of short FDLs, the burst-loss probability can be approximated by

$$
\beta \approx \operatorname{Pr}\left\{A_{W}, W>B\right\} .
$$

Therefore, we shall focus on the evaluation of $\operatorname{Pr}\left\{A_{W}, W>\right.$ $B\}$.

Accordingly, we can ignore the FDL reservation phase and assume that an FDL is always available when it is needed. In this context, an incoming burst arriving at time $t$ can enter a wavelength in an FDL if there is at least one wavelength available, and the waiting time of this burst is less than $B$; otherwise, the burst is blocked. This behavior can be captured by the balking queueing model, which is a special case of a queue with impatience [14]. In queueing models with balking, an arriving customer joins the system only if the expected waiting time is less than a given threshold. Based on the above argument, we can model the dynamics of optical-burst switching with FDLs as an $M / M / k$ queue with balking.

We shall take the state of the system to be the number of bursts in the system. Let $\lambda_{i}$ and $\mu_{i}, i \geq 0$, be the burst-generation rate and the burst-service rate, respectively, when the system state is $i$. For classless traffic, $\mu_{i}=\min \{i, k\} \mu$, since there are $k$ wavelengths available in the output port. For $i<k$, there is no burst loss, since in this case, the incoming DB can always find an idle channel to carry it. Therefore, $\lambda_{i}=\lambda$ for $i<k$. However, under the LAUC scheduling algorithm, when all channels are busy, i.e., $i \geq k$, a burst is lost if the earliest available time of all wavelengths is greater than $t+B$. This behavior can be modeled approximately by assuming that the burst is lost if the number of service completions within the time duration $B$ is less than $i-k+1$.

Let $\beta_{i}$ be the burst-loss probability when the system is in state $i$. We have $\lambda_{i}=\lambda\left(1-\beta_{i}\right)$. Clearly, $\beta_{i}=0$ for $i<k$. For $i \geq k$, the probability of no loss in state $i, 1-\beta_{i}$, is equal to the 
probability that there are at least $i-k+1$ service completions within time $B$. Thus, we have (cf. [14])

$$
\begin{aligned}
\beta_{i} & =1-\operatorname{Pr}[i-k+1 \text { completions within time } B] \\
& =1-\int_{0}^{B} e^{-k \mu x} \frac{k \mu(k \mu x)^{i-k}}{(i-k) !} d x \\
& =1-\sum_{j=0}^{i-k} e^{-k \mu B} \frac{(k \mu B)^{j}}{j !} .
\end{aligned}
$$

Having specified $\lambda_{i}$ and $\mu_{i}$, one easily obtains the steady-state probabilities $\pi_{i}$ that the system is in state $i, i \geq 0$

$$
\begin{aligned}
& \pi_{0}=\left[1+\sum_{j=0}^{\infty} \prod_{i=0}^{j-1} \frac{\lambda_{i}}{\mu_{i+1}}\right]^{-1} \\
& \pi_{i}=\pi_{0} \prod_{j=0}^{i-1} \frac{\lambda_{j}}{\mu_{j+1}}, \quad i \geq 1
\end{aligned}
$$

Thus, in the regime of short FDLs, the overall burst-loss probability is simply given by

$$
\beta_{S}=\sum_{j=k}^{\infty} \pi_{j} \beta_{j} \triangleq \operatorname{BALK}(k, \rho, F, \mu B),
$$

where we have introduced the notation $\operatorname{BALK}(k, \rho, F, \mu B)$ to represent the loss probability under the balking model as a function of the parameters $k, \rho, F$, and $\mu B$, which is the maximum FDL delay $B$ normalized by the mean burst duration $1 / \mu$.

\section{B. $M / M / k / k+m$ Model for Long FDLs}

In the regime of long FDLs, i.e., $B \gg 0$, it is more likely for a burst to be blocked due to lack of available FDLs than for the burst to be blocked because the waiting time $W$ is greater than $B$. This suggests that in the regime of long FDLs, the burst-loss probability can be approximated by

$$
\beta_{L} \approx \operatorname{Pr}\left\{A_{W}, A_{F}\right\}
$$

Therefore, we shall focus on the evaluation of $\operatorname{Pr}\left\{A_{W}, A_{F}\right\}$, which is actually equal to the burst-loss probability in the asymptotic regime, where $B \rightarrow \infty$.

In the regime of asymptotically long FDLs, each virtual buffer can provide any delay that might be required in order to schedule an incoming DB. Since there are $k$ wavelength channels and $m=k F$ virtual buffers, the buffer behavior can be approximated by an $M / M / k / k+m$ queueing model. This is precisely the approximate model for FDLs proposed earlier in [1] and [3]. Using the $M / M / k / k+m$ model, the burst-loss probability in the regime of long FDLs is given approximately by

$$
\beta_{L}=\frac{\rho^{k+m} /\left(k^{m} \cdot k !\right)}{\sum_{j=0}^{k-1} r^{j} / j !+\sum_{j=k}^{k+m} \rho^{j} /\left(k^{j-k} \cdot k !\right)} .
$$

We introduce the notation QUEUE $(k, \rho, F) \triangleq \beta_{L}$, as defined in (16), to represent the burst-loss probability under the $M / M / k / k+m$ model as a function of the parameters $k, \rho$, and $F$.

\section{Overall Approximate Model}

In the regime of short FDLs, we have $\beta_{S} \approx b_{W}$, while in the regime of long FDLs, we have $\beta_{L} \approx b_{F}$. Moreover, when $B$

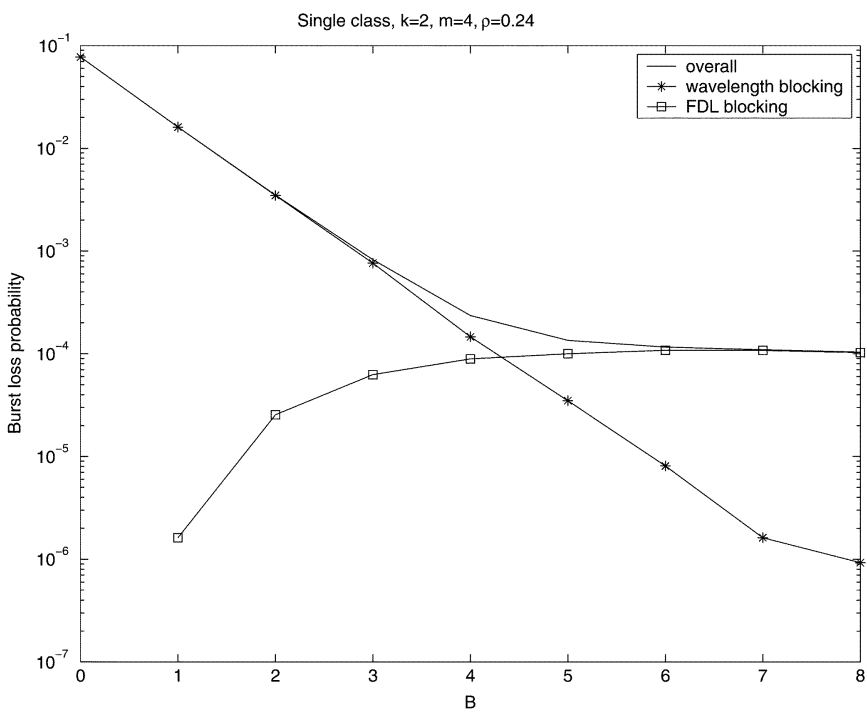

Fig. 4. Validation of the asymptotic approximation model by computer simulation.

is small, we have already remarked that $b_{W} \gg b_{F}$. Similarly, when $B$ is large, $b_{F} \gg b_{W}$. Therefore, a reasonable approximation for the overall burst-loss probability is

$$
\beta \approx \beta_{S}+\beta_{L}
$$

Fig. 4 shows results obtained from computer simulation for the probability $\beta_{W}$ of wavelength blocking, and the probability $\beta_{F}$ of FDL blocking, as functions of the maximum FDL length $B$. The overall burst-loss probability is also shown. The figure provides empirical evidence of the validity of (17). Numerical validation of the approximation (17) is discussed further in Section VI.

\section{IMPACT OF FDL DELAY GRANULARITY}

As discussed in Section II, a variable-delay FDL can only assume a finite set of values between 0 and $B$, i.e., multiples of a unit delay value $b$. This issue was studied by Callegati in [10] by applying the balking model to evaluate the burst-loss probability. We shall follow Callegati's approach in extending the overall approximate model of Section IV to incorporate the impact of the unit delay granularity $b$.

Suppose that upon arrival to the switch at time $T_{s}$, a DB must wait a minimum waiting time of $W$ until an outgoing wavelength channel becomes free. In the idealized variable-delay FDL model of Section III, the DB would be delayed by an amount $W$. However, due to the granularity $b$ of the variabledelay FDL, the burst will actually be delayed by an amount

$$
\tau_{e}=\left\lceil\frac{W}{b}\right\rceil b \text {. }
$$

Consequently, the wavelength channel will be idle from time $T_{s}+W$ to time $T_{s}+\Delta$ for a duration $\tau=\tau_{e}-W$, until transmission of the burst ensues. This effect can be modeled as an artificial increase in the burst length [10]. Under the assumption that the arrival process is independent of the state of the optical buffer and the lengths of bursts are independent, $\tau$ will be uniformly distributed over the interval $[0, b]$. 
Let $\bar{S}$ denote the mean length of a burst. We shall let $\bar{S}_{e}$ denote the mean length of the extended burst. Let $\pi_{d}$ denote the probability that an incoming burst will be delayed. Then we have

$$
\bar{S}_{e}=\left(1-\pi_{d}\right) \bar{S}+\pi_{d}(\bar{S}+b / 2)=\bar{S}+\pi_{d} b / 2 .
$$

The traffic load to the system is given by $\rho=\lambda / \mu=\lambda \bar{S}$. The effective load due to the FDL granularity $b$ is given by

$$
\rho_{e}=\lambda \bar{S}_{e} .
$$

Although the distribution of the burst length $S$ is exponential, the distribution of $S_{e}$ is not. Nevertheless, to obtain a tractable approximation, we shall assume that $S_{e}$ is exponentially distributed (cf. [10]). Given an initial value for $\bar{S}_{e}$, an iterative procedure can be used to obtain an approximate value for $\bar{S}_{e}$.

More precisely, $\bar{S}_{e}$ is initialized to $\bar{S}$. The extension to the Markovian model of Section III is obtained by iteratively solving the model, with each successive iteration starting from the assumption that the mean burst length is given by the current value of $\bar{S}_{e}$. From the solution of the Markovian model, the probability $\pi_{d}$ of a burst being delayed can be obtained. Then a new value of $\bar{S}_{e}$ can be computed from (19). The process is repeated until the error between successive approximations of $\bar{S}_{e}$ is less than some prescribed level of accuracy $\epsilon$. From the final value of $\bar{S}_{e}$, the Markovian model is applied once more to obtain an approximation for the blocking probability $\beta_{e}$, taking into account the effect of FDL granularity. In a similar way, the asymptotic approximations for burst-loss probability discussed in Section IV can be extended to incorporate the effect of FDL granularity.

\section{NUMERICAL RESULTS}

We constructed a discrete event-driven simulator based on the Dartmouth Scalable Simulation Framework (DaSSF) [15] to verify the accuracy of our analytical results. In each simulation trial, we discarded the data generated during the first $10^{5} \mathrm{~s}$ to eliminate transient effects [16]. Each trial lasted $10^{7}$ s. 30 trials were run, in total, to obtain the statistics of interest. All simulation results are shown with $95 \%$ confidence intervals. Unless specified otherwise, the mean burst length $1 / \mu$ is assumed to be set to one, so that the parameter $B$ represents the normalized maximum FDL length.

Fig. 5 plots the burst-loss probability versus the utilization $\rho$ for different values of the parameter $B$, when $k=2$ and $F=3$. The curves denoted by "Analysis" were obtained using the Markovian model discussed in Section III and show close agreement with the curves obtained by simulation. Observe that the performance gain achievable by using longer FDLs is more pronounced when the utilization is low.

When $k>2$, solving the Markovian model becomes computationally expensive, so we resort to the asymptotic approximation of Section IV. Fig. 6 plots performance curves for a system with $k=8, F=2$, and $\rho=0.8$. Here, the curve denoted by "Analysis" is obtained from the asymptotic overall approximation for burst-loss probability. Observe that the asymptotic approximation follows the simulation curve reasonably well. The corresponding curves obtained from the approximation of Yoo et al. [3] and Fan et al. [9] are shown for comparison. The approximation of Yoo et al. [3] is represented by the horizontal

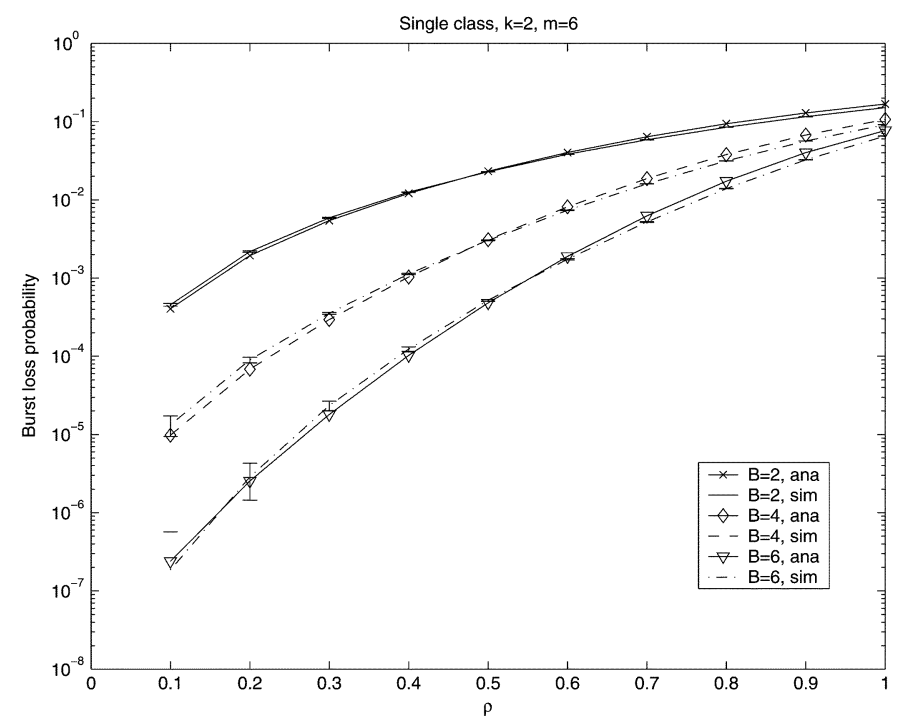

Fig. 5. Burst-loss probability as a function of utilization.

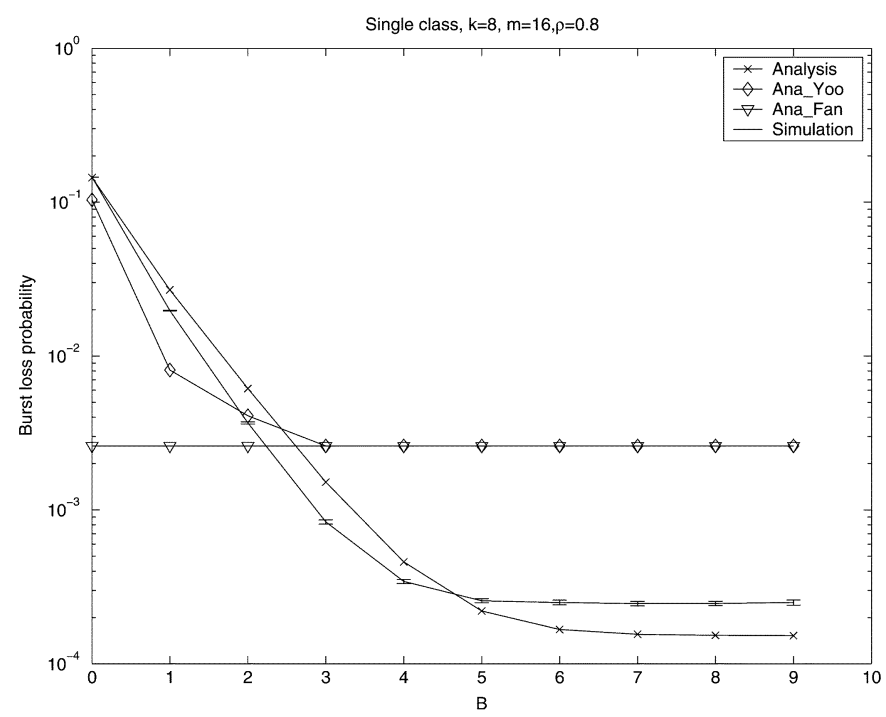

Fig. 6. Burst-loss probability as a function of maximum FDL delay.

line between $10^{-3}$ and $2\left(10^{-3}\right)$. This approximation does not follow the decreasing trend of the burst-loss probability with increasing maximum FDL value, and is accurate only for values in the neighborhood of $B=3$. The approximation discussed by Fan et al. improves upon the Yoo approximation, but still significantly underestimates the burst-loss probability when $B<2$, and overestimates the burst-loss probability when $B>3$.

In Fig. 7, the burst-loss probability obtained using the asymptotic approximation is plotted versus the number of wavelengths $k$ per fiber, in a system with two fibers for different values of the parameter $B$. In this system, each input/output port consists of two fibers, with each fiber containing $k$ wavelength channels for DB transmission. The case $B=0$ is equivalent to an OBS system without optical buffering. The figure clearly shows that by employing FDLs, the gain in burst-loss performance becomes more pronounced as the number of wavelengths in the system is increased.

Fig. 8 illustrates the effect of the FDL unit delay granularity. Here, the burst-loss probability is plotted as a function of the 


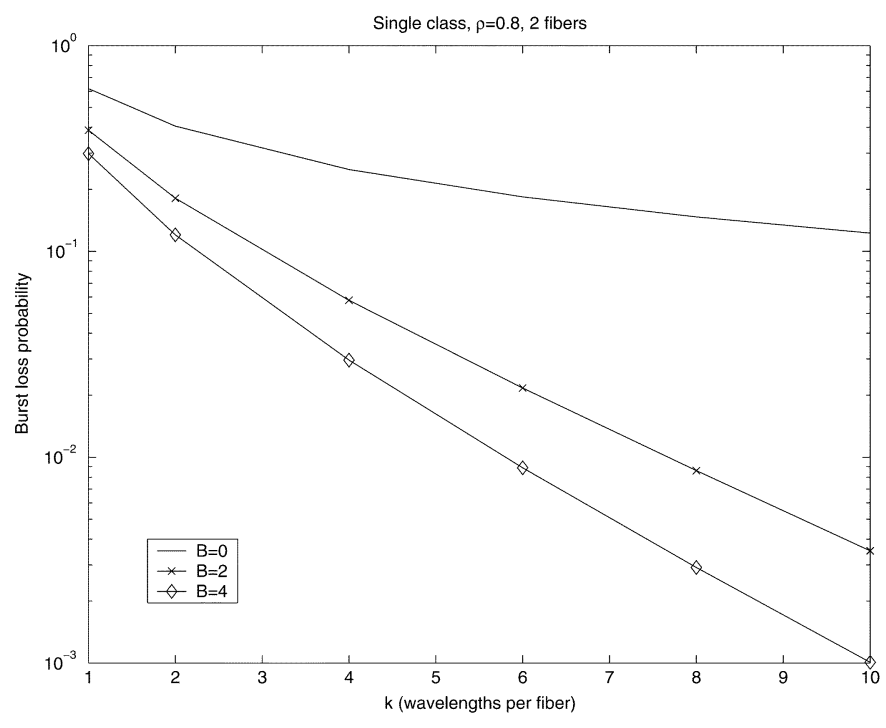

Fig. 7. Burst-loss probability as a function of number of wavelengths.

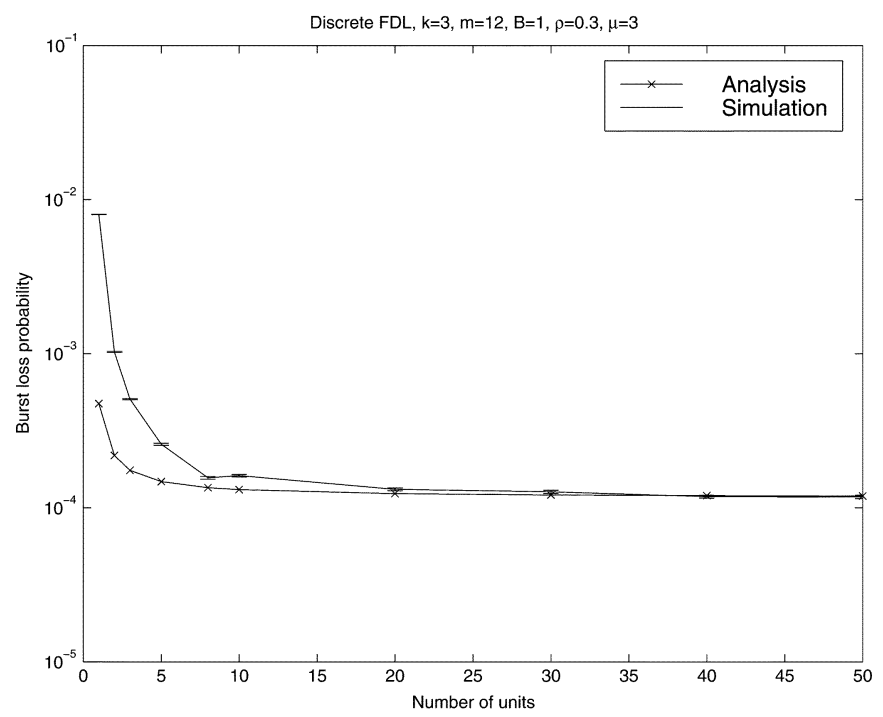

Fig. 8. Burst-loss probability as a function of unit FDL delay granularity.

number of delay units for a constant maximum FDL delay value of $B=1$. The other parameters are set as follows: $\mu=3, k=3$, and $F=4$ (so that $m=12$ ), and $\rho=0.3$. For each value $x$ on the horizontal axis, the value of the unit delay is set to $b=B / x=1 / x$. The analysis curve was obtained using the extension to the asymptotic approximation discussed in Section V, and is in close agreement with the simulation curve. Observe that the curve is nearly flat when the number of delay units is greater than eight. This implies that when a sufficient number of delay units is employed (in this case, eight), the performance of the variable-delay FDL buffer with discrete delay units is nearly the same as that of the ideal variable-delay FDL model assumed in Section III.

\section{CONCLUSION}

We have developed a new model to evaluate the performance of an OBS with FDLs. The model takes the form of a multidimensional Markov chain that is very accurate under the assumptions of Poisson burst arrivals and exponentially distributed burst lengths. We have also developed approximations of the Markovian model that can be computed efficiently and yield results comparing favorably with results from simulation. The analytical model and approximations yield important insights into the deterministic delay and balking characteristics of OBS system performance over the entire range of FDL lengths, particularly in the regimes of short and long FDLs. We have seen that the use of FDLs can significantly reduce the burst-loss probability. The system performance improves as the length of the FDL increases, but the gains diminish quickly after a certain point. Our approximations are simple and accurate enough to be used for dimensioning FDLs in OBS architectures.

We extended our model and approximations to incorporate the impact of unit FDL granularity on OBS performances by adapting an iterative approach proposed by Callegati [10]. Our numerical results suggest that only a small number of unit FDL delays is needed to achieve the performance of the idealized variable-delay FDL upon which our original analytical model was based. This provides some justification for basing our original analysis on the idealized variable-delay FDL model.

An interesting open problem is to develop accurate models and approximations for optical-burst switching with differentiated service classes. Toward that end, Yoo et al. [3] proposed a prioritized OBS scheme and applied a conservation law to obtain approximate blocking probabilities. By applying this conservation law, the more accurate models developed in this paper can also be extended to incorporate the prioritized OBS scheme. However, this approach tends to give inaccurate blocking probability estimates for lower priority traffic classes. A more finegrained analysis of the interclass contention and its effect on the overall burst-loss probability is needed, in order to obtain more accurate results for the performance of low-priority traffic.

\section{REFERENCES}

[1] J. Turner, "Terabit burst switching," J. High Speed Networks, vol. 8, pp. 3-16, Jan. 1999.

[2] C. Qiao and M. Yoo, "Optical burst switching (OBS)-A new paradigm for an optical internet," J. High Speed Networks, vol. 8, pp. 69-84, Jan. 1999.

[3] M. Yoo, C. Qiao, and S. Dixit, "QoS performance of optical burst switching in IP over WDM networks," IEEE J. Select. Areas Commun., vol. 18, pp. 2062-2071, Oct. 2000.

[4] Y. Xiong, M. Vandenhoute, and H. Cankaya, "Control architecture in optical burst-switched WDM networks," IEEE J. Select. Areas Commun., vol. 18, pp. 1838-1851, Oct. 2000.

[5] I. Baldine, G. Rouskas, H. Perros, and D. Stevenson, "JumpStart: A just-in-time signaling architecture for WDM burst-switched networks," IEEE Commun. Mag., vol. 40, pp. 82-89, Feb. 2002.

[6] S. Amstutz, "Burst switching-An introduction," IEEE Commun. Mag., vol. 21, pp. 36-42, Aug. 1983.

[7] X. Lu and B. L. Mark, "Analytical modeling of optical burst switching with fiber delay lines," in Proc. IEEE/ACM Int. Symp. Modeling, Analysis, Simulation of Computer, Telecommunications Systems, Fort Worth, TX, Oct. 2002, pp. 501-506.

[8] - "A new performance model of optical burst switching with fiber delay lines," in Proc. IEEE Int. Conf. Communications, vol. 2, Anchorage, AK, May 2003, pp. 1365-1369.

[9] P. Fan, C. Feng, Y. Wang, and N. Ge, "Investigation of the time-offsetbased QoS support with optical burst switching in WDM networks," in Proc. IEEE Int. Conf. Communications, 2002, pp. 2682-2686.

[10] F. Callegati, "Optical buffers for variable-length packets," IEEE Commun. Lett., vol. 4, pp. 292-294, Sept. 2000.

[11] D. Hunter, M. Chia, and I. Andonovic, "Buffering in optical packet switches," J. Lightwave Technol., vol. 16, pp. 2081-2094, Dec. 1998. 
[12] R. Ramaswami and K. Sivarajan, Optical Networks: A Practical Perspective, 2nd ed. San Francisco, CA: Morgan Kaufmann, 2002.

[13] C. Murthy and M. Gurusamy, WDM Optical Networks: Concepts, Design, and Algorithms. Englewood Cliffs, NJ: Prentice-Hall, 2002.

[14] D. Gross and C. Harris, Fundamentals of Queueing Theory, 3rd ed. New York: Wiley, 1998.

[15] J. Liu, D. M. Nicol, B. Premore, and A. Poplawski, "Performance prediction of a parallel simulator," in Proc. Workshop Parallel, Distributed Simulation, Atlanta, GA, May 1999.

[16] A. Law and W. D. Kelton, Simulation Modeling and Analysis. Boston, MA: McGraw-Hill, 2000.

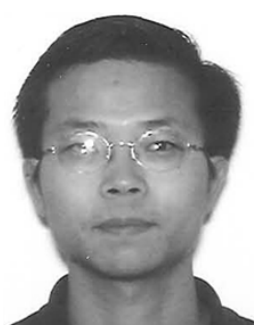

Xiaomin Lu (S'00) received the B.E. and M.E. degrees from the University of Science and Technology of China (USTC), Hefei, China, in 1994 and 1997, respectively, and the Ph.D. degree in electrical and computer engineering from George Mason University, Fairfax, VA, in 2002.

Between the years 2000 and 2002, he was a Graduate Research Assistant in the Department of Electrical and Computer Engineering at George Mason University. His research interests include high-speed networks, broadband switch architectures, stochastic modeling, and wire-speed packet processing using network processors.

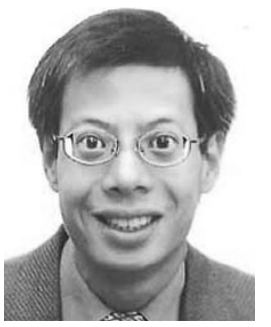

Brian L. Mark (M'91) received the B.A.Sc. degree in computer engineering with an option in mathematics from the University of Waterloo, Waterloo, ON, Canada, in 1991, and the Ph.D. degree in electrical engineering from Princeton University, Princeton, NJ, in 1995.

He was a Research Staff Member at the C\&C Research Laboratories, NEC USA, from 1995 to 1999. In 1999, he was on part-time leave from NEC as a Visiting Researcher at Ecole Nationale Supérieure des Télécommunications, Paris, France. In 2000, he joined the Department of Electrical and Computer Engineering, George Mason University, Fairfax, VA, where he is currently an Assistant Professor. His main research interests lie broadly in the design, modeling, and analysis of communication systems, computer systems, and communication networks.

Dr. Mark was a co-recipient of the best conference paper award for IEEE Infocom'97. He received a National Science Foundation CAREER Award in 2002. 